\title{
Piezoelectric effect in p-Si/SiGe/(001)Si modulation doped heterostructures
}

\author{
V.K.Dugaev ${ }^{1}$, O.A.Mironov ${ }^{2}$; S.V.Kosyachenko ${ }^{3}$ \\ 1 Chernivtsi Branch of the Institute of Materials Science Problems, \\ National Academy of Sciences of Ukraine, \\ 5 Vilde Str., 58001 Chernivtsi, Ukraine \\ 2 Department of Physics, University of Warwick, Coventry, CV4 7AL, UK \\ 3 Trade and Economy Institute of Chernivtsi, \\ 1 Central Square, 58000 Chernivtsi, Ukraine
}

Received April 17, 2000, in final form October 31, 2000

\begin{abstract}
We present the results of calculations of the piezoelectric effect in a Si/SiGe multilayer structure with a narrow quantum well and a wide layer of doped $\mathrm{Si}$ semiconductor. The proposed theory is a possible explanation of some recent experiments on these structures.
\end{abstract}

Key words: SiGe heterostructures, piezoelectricity

PACS: 77.65.Ly, 85.50.+k, 73.61.-r

\section{Introduction}

The quantum structures with $\mathrm{Si} / \mathrm{Si}_{1-x} \mathrm{Ge}_{x}$ layers, realized as superlattices, quantum wells (QW) and heterojunctions have been intensively studied recently because of their extreme technological importance. It is mostly related with huge industrial productions of Si-based materials together with their numerous applications. The $\mathrm{Si} /$ SiGe-based structures and superlattices can be used as components of faster microchips, integrated optoelectronic devices, and many others. The combination of controlled variation of the composition, strain, and thickness of the layers provides better electronic and optical properties of the structures as compared to any bulk semiconductor $[1,2]$.

There exists a well-known problem in the theoretical modelling of certain properties of the semiconducting multilayered structures. The point is that the energy diagram (spatial variation of the potential) depends on a number of factors such as real distribution of electrons and holes, and doping profile as well as on the distribu-

\footnotetext{
*On leave from Usikov's Institute for Radiophysics and Electronics, National Academy of Sciences of Ukraine, 310085 Kharkiv, Ukraine
} 
tion of deformations of the crystal lattice. Besides, electron and hole eigenfunctions and eigenvalues are determined by the electrostatic potential and deformation profiles. From the theoretical point of view it leads to a rather complicated problem of self-consistent solution of Poisson's equation together with Schroedinger's and elasticity theory's equations.

The main source of deformations in a multilayer structure is non-compatibility of crystal lattice of constituents. In the case of the non-centro-symmetrical crystals like, e.g., III-V compounds, there are also deformations produced by the piezoelectric effect due to internal electric fields accompanying the potential relief. Recent investigations of piezoelectric coupling in the AlGaN/InGaN heterostructures [4] give evidence of its strong effect on the properties of the electron gas in these systems.

It is commonly believed that the piezoelectric effect is absent in Si-based structures since piezoelectric tensor is equal to zero in the corresponding bulk crystals. On the other hand the crystallic symmetry is changed with the creation of quantum structures. That change may lead to a nonvanishing nonuniform piezoelectricity.

Recently it was discovered experimentally [5-7] that when a pulsed electric field is applied to a $\mathrm{Si} / \mathrm{SiGe}$ structure with an electric-field vector $\vec{E}$ along the growth direction, the deformation waves appear in such structures. It clearly demonstrates the existence of the piezoelectricity. Up till now the nature of this effect in the structures with separate QW is not quite well understood.

The absence of the piezoelectric effect in bulk $\mathrm{Si}$ or $\mathrm{Si}_{1-x} \mathrm{Ge}_{x}$ solid solutions is due to their symmetry [8]: the corresponding crystallic class $O_{h}$ contains the center of the inversion. It becomes clear from the same considerations that the non-zero components of the piezoelectric tensor $\gamma_{i, j k}$ can arise if a distinguished direction arises in the structure.

In the case of a structure with symmetrical Si/SiGe/Si QW (QW for holes being located on the SiGe solid solution region) there is a distinguished axis perpendicular to the QW plane, but there is no any definite direction of this axis. Thus, in the average, piezoelectric tensor has to vanish in this case. The situation changes when a nonuniform doping is used, as it was in the case of the cited experiments [5-7], see figure 1. Indeed, if the $x$ axis is chosen along [001] direction, the symmetry class changes from $O_{h}$ to $C_{4 v}$, in which case $\gamma_{x, y y}=\gamma_{x, z z}, \gamma_{y, y x}=\gamma_{z, z x}$, and the $\gamma_{x, x x}$ components can be nonzero. Obviously, these components should depend on $x$, so that the maximum value is reached in a vicinity of the QW vanishing deep in the bulk of Si and SiGe. The physical nature of the piezoelectric effect becomes even more clear if we keep in mind a simple model with separated charges where on the one side there is the QW with a number of carriers (holes, in case of $p$-doping, as shown in figure 1), and on the other side there is a layer of uncompensated charged acceptors. Consequently, when an AC electric field $\vec{E}$ is applied along $x$, the covers of this "capacitor" will be moving, thus exciting the acoustic waves. 


\begin{tabular}{|r|}
\hline $\mathrm{p}-\mathrm{Si}\left(\mathrm{p}=2.5^{*} 10^{18} \mathrm{~cm}^{-3}\right) 30 \mathrm{~nm}$ \\
\hline $\mathrm{i}-\mathrm{Si}$ (spacer) $20 \mathrm{~nm}$ \\
\hline$++++\mathrm{p}=1.8^{*} 10^{11} \mathrm{~cm}^{-3}++++$ \\
\hline SiGe $30 \mathrm{~nm}$ \\
\hline $\mathrm{i}-\mathrm{Si}$ (buffer) $300 \mathrm{~nm}$ \\
\\
$\mathrm{n}-\mathrm{Si}$ (substrate) $0.57 \mathrm{~mm}$ \\
\end{tabular}

Figure 1. Schematical picture of the $\mathrm{Si} / \mathrm{SiGe}$ heterostructure.

\section{Model and results of calculations}

The energy diagram illustrating the position of valence band edge and the filling with holes is presented schematically in figure 2, for a structure consisting of the QW and some layer inside Si doped with acceptors. The size-quantized levels are located within QW near the Si/SiGe interface.

Let us consider a simplified model (figure 3), in which the wide doped region of width $d$ is located at a distance $b$ (spacer width) from the QW with only one sizequantization level below the Fermi energy $\mu$ by $\varepsilon_{0}$. For simplicity, from now on we deal with a model with free electrons instead of the holes. Correspondingly, we suppose that the region $d$ is doped with donor impurities. Let the average concentration of the donors be equal to $n_{0}$.

The two-dimensional layer of electrons, trapped into QW, can be described by a distribution of electrons $\nu \delta(x-d-b)$, where $\nu$ is the two-dimensional density of electrons. The Poisson's equation for the electrostatic potential $\varphi(x)$ in the region 


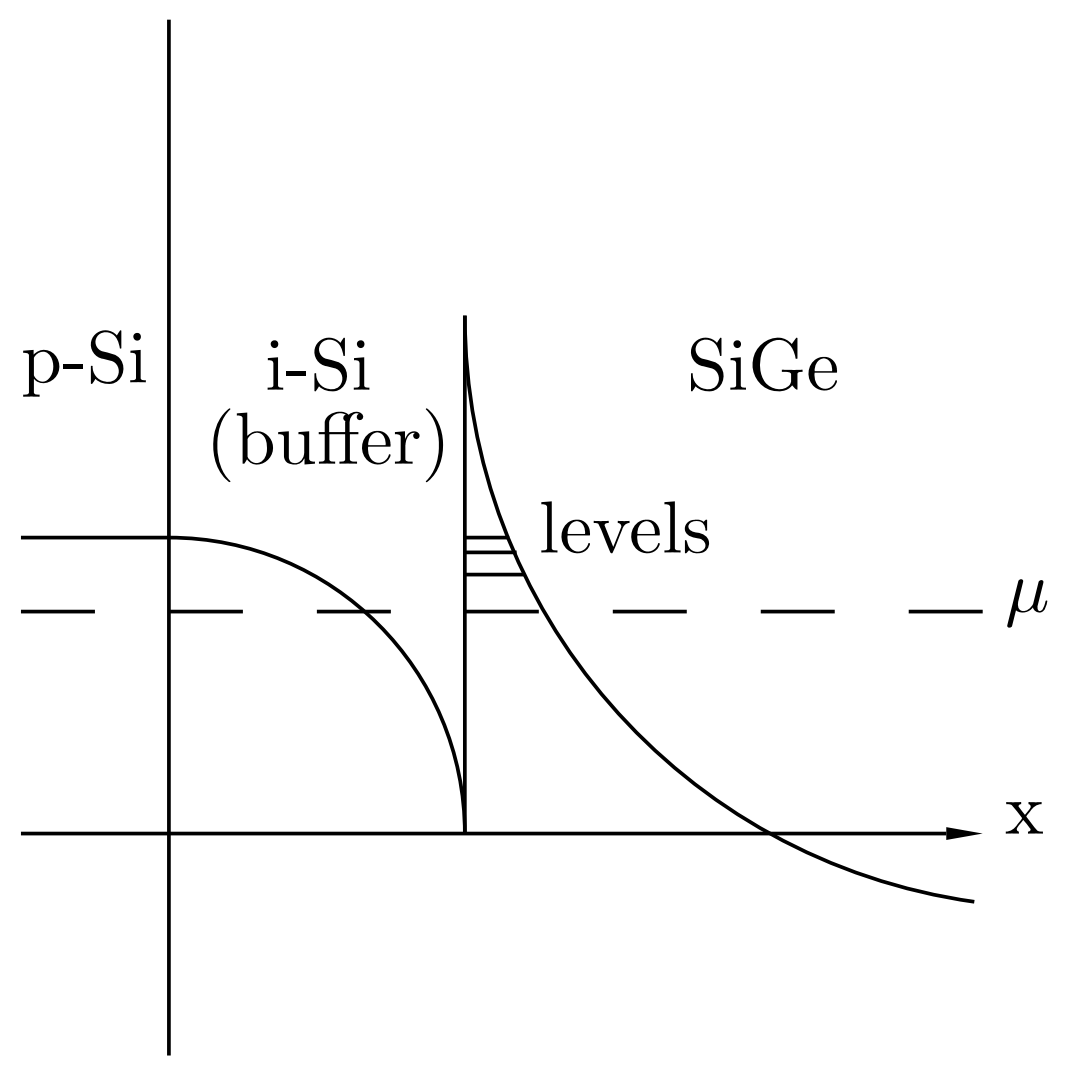

Figure 2. Energy diagram of the $\mathrm{Si} / \mathrm{SiGe}$ heterostructure.

$0<x<d$ is

$$
\frac{\mathrm{d}^{2} \varphi}{\mathrm{d} x^{2}}=-\frac{4 \pi e}{\varepsilon}\left[n(x)-n_{0}\right]
$$

where $n(x)$ is the distribution of free electrons at $0<x<d$ and $\varepsilon$ is the dielectric constant.

Within the semiclassical approximation for the electronic gas at $0<x<d$, the density $n(x)$ and the electrostatic potential $\varphi(x)$ are related by (we put $\hbar=1$ )

$$
\left[3 \pi^{2} n(x)\right]^{2 / 3}=2 m^{*}[\mu-e \varphi(x)],
$$

where $m^{*}$ is the electron effective mass. The semiclassical approximation is justified due to the large value of $d$ as compared to the electron wavelength $\lambda$. Due to the electrical neutrality, the total number of the electrons inside the doping region and the localized electrons trapped into the QW, is constant

$$
\int_{0}^{d} n(x) \mathrm{d} x+\nu=n_{0} d .
$$

We assume that the deviation of concentration from the equilibrium $\delta n(x)=$ $n(x)-n_{0}$ is small, $\delta n(x) \ll n_{0}$. Then the solution of equations (1) and (2) has the form

$$
\varphi(x)=\varphi_{1} \mathrm{e}^{-x / L_{\mathrm{c}}}+\varphi_{2} \mathrm{e}^{x / L_{\mathrm{c}}}
$$




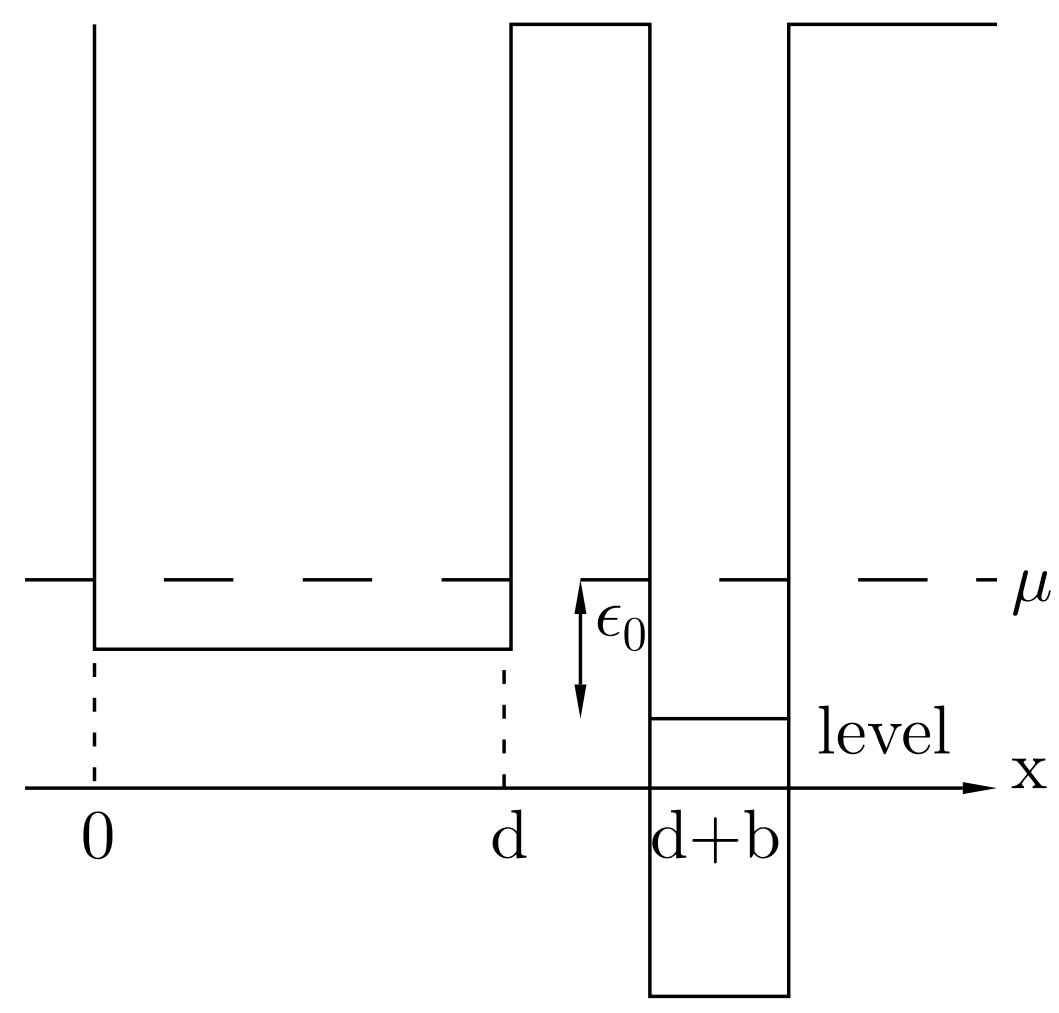

Figure 3. Simplified model of a structure with narrow quantum well.

$$
\delta n(x)=-\frac{e m^{*} k_{\mathrm{F}}}{\pi^{2}}\left(\varphi_{1} \mathrm{e}^{-x / L_{\mathrm{c}}}+\varphi_{2} \mathrm{e}^{x / L_{\mathrm{c}}}\right),
$$

where $k_{\mathrm{F}}$ is the Fermi momentum, $L_{\mathrm{c}}$ is the screening length in the doped region

$$
L_{\mathrm{c}}=\left(\varepsilon \pi / 4 k_{\mathrm{F}} m^{*} e^{2}\right)^{1 / 2},
$$

and $\varphi_{1}, \varphi_{2}$ are certain constants. Using equation (3), we can find a relation between $\varphi_{1}$ and $\varphi_{2}$

$$
\varphi_{2}=\frac{1}{\exp \left(d / L_{\mathrm{c}}\right)-1}\left[\frac{\pi^{2} \nu}{e m^{*} k_{\mathrm{F}} L_{\mathrm{c}}}-\varphi_{1}\left(1-\exp \left(-\frac{d}{L_{\mathrm{c}}}\right)\right)\right] .
$$

Let us find the variation of potential $\varphi(x)$ at $x<0$ and $x>d$ (figure 4). The Poisson's equation (1) has a nonzero right-hand side for $x=d+b$

$$
\frac{\mathrm{d}^{2} \varphi}{\mathrm{d} x^{2}}=-\frac{4 \pi e}{\varepsilon} \nu \delta(x-d-b)
$$

and vanishes for $x>d$ and $x<0$ (we neglect a small nonzero carrier concentration in these regions). Let the external field $\vec{E}$ be applied along the $x$ axis. Then we can write

$$
\varphi(x)=\left\{\begin{array}{cc}
-E x+A, & x<0 \\
\varphi_{1} \exp \left(-x / L_{\mathrm{c}}\right)+\varphi_{2} \exp \left(x / L_{\mathrm{c}}\right), & 0<x<d \\
B x+C, & d<x<d+b \\
-E x+D, & x>d+b
\end{array}\right.
$$




\section{$\varphi(\mathrm{x})$}

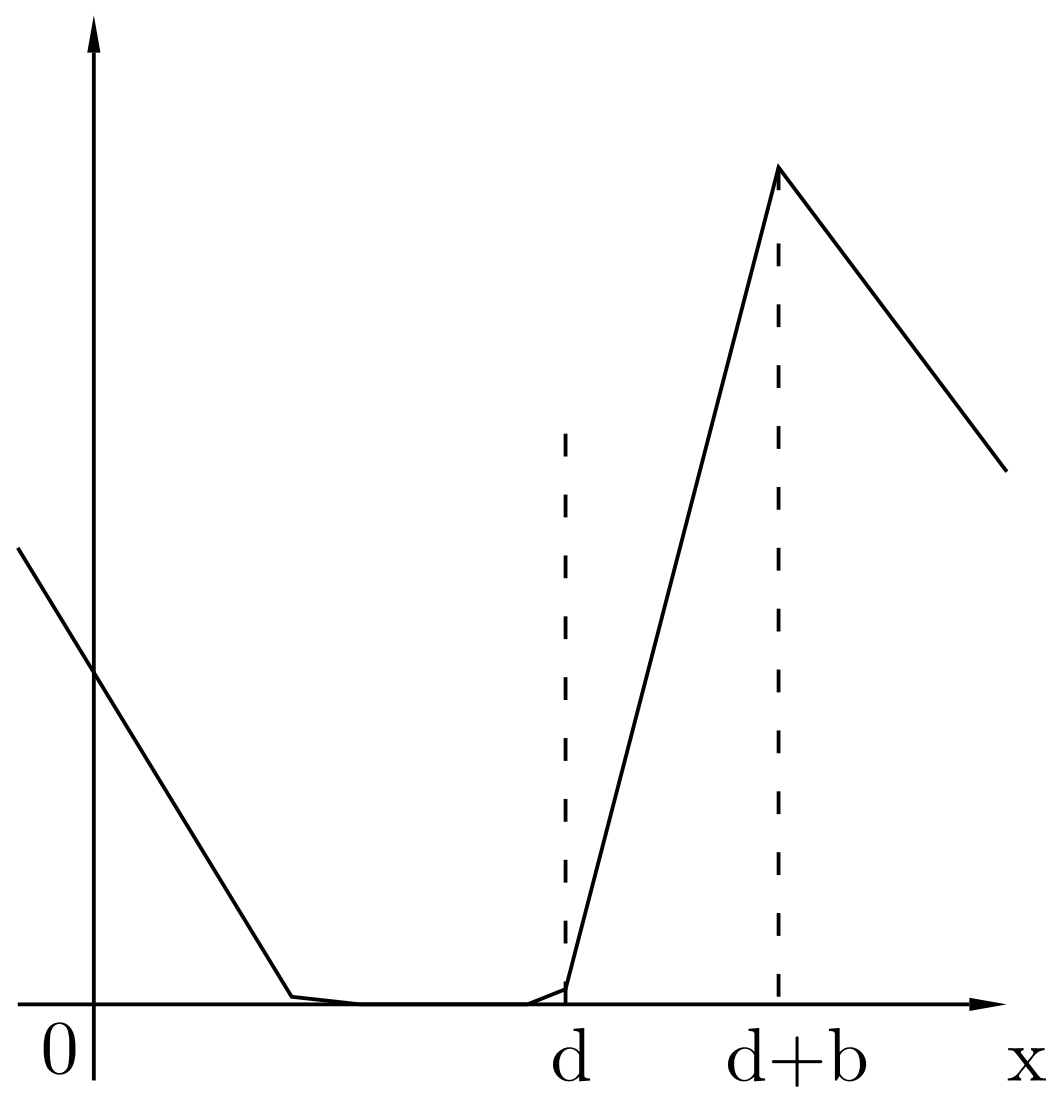

Figure 4. Variation of electrostatic potential for the model heterostructure with external electric field.

where $A, B, C, D$ are constants, which should be determined from the conditions of matching at $x=0, x=d$, and $x=d+b$.

The equation determining a charge density in QW for nonzero potential $\varphi_{d+b} \equiv$ $\varphi(d+b)$ is given by

$$
\nu=\frac{m^{*}}{\pi}\left(\mu+\varepsilon_{0}-e \varphi_{d+b}\right) .
$$

With the aid of equations (4)-(9), and matching $\varphi(x)$ at the interfaces $x=0, x=d$, and $x=d+b$, we find

$$
\begin{aligned}
\varphi_{1} & =\frac{1}{2 \sinh \left(d / L_{\mathrm{c}}\right)}\left[E L_{\mathrm{c}}\left(\exp \frac{d}{L_{\mathrm{c}}}-1\right)+\frac{\pi^{2} \nu}{e m k_{\mathrm{F}} L_{\mathrm{c}}}\right] \\
\varphi_{2} & =\frac{1}{2 \sinh \left(d / L_{\mathrm{c}}\right)}\left[-E L_{\mathrm{c}}\left(1-\exp \left(-\frac{d}{L_{\mathrm{c}}}\right)\right)+\frac{\pi^{2} \nu}{e m k_{\mathrm{F}} L_{\mathrm{c}}}\right] \\
\nu & =\rho_{0}\left[\mu+\varepsilon_{0}+e E\left(b-L_{\mathrm{c}} \frac{1-\cosh \left(d / L_{\mathrm{c}}\right)}{\sinh \left(d / L_{\mathrm{c}}\right)}\right)\right]\left[1+\frac{4 b}{a_{\mathrm{B}}}+\frac{\pi}{k_{\mathrm{F}} L_{\mathrm{c}}} \operatorname{coth}\left(d / L_{\mathrm{c}}\right)\right]^{-1},
\end{aligned}
$$

where $\rho_{0}=m^{*} / \pi$ is the two-dimensional density of states, $\mu=\left(3 \pi^{2} n_{0}\right)^{2 / 3} / 2 m^{*}$, and 
$a_{\mathrm{B}}=\varepsilon / m^{*} e^{2}$ is the effective Bohr radius.

Using (4), (10)-(12), we can find the distribution of electric field $E(x)$ inside the region $0<x<d$ (in fact, only a part of it depending on the external electric field will be taken into account here, see below)

$$
\begin{aligned}
E(x)=-\frac{\mathrm{d} \varphi}{\mathrm{d} x}= & -\frac{E}{\sinh \left(d / L_{\mathrm{c}}\right)}\left(\sinh \frac{d-x}{L_{\mathrm{c}}}+\sinh \frac{x}{L_{\mathrm{c}}}\right. \\
& \left.-\zeta \frac{\left(b / L_{\mathrm{c}}\right) \sinh \left(d / L_{\mathrm{c}}\right)-1+\cosh \left(d / L_{\mathrm{c}}\right)}{\sinh \left(d / L_{\mathrm{c}}\right)\left[1+\left(4 b / a_{\mathrm{B}}\right)+\varsigma \operatorname{coth}\left(d / L_{\mathrm{c}}\right)\right]} \sinh \frac{x}{L_{\mathrm{c}}}\right),
\end{aligned}
$$

where the notation $\zeta=\pi / k_{\mathrm{F}} L_{\mathrm{c}}$ is used.

Using equation (5), the distribution of charge density $q(x)$ in $0<x<d$ region (only a part independent on $E$ ) can be found

$$
q(x)=-e \cdot \delta n(x)=\frac{e \rho_{0} \cosh \left(x / L_{\mathrm{c}}\right)}{L_{\mathrm{c}} \sinh \left(d / L_{\mathrm{c}}\right)} \cdot \frac{\mu+\varepsilon_{0}}{1+\left(4 b / a_{\mathrm{B}}\right)+\varsigma \operatorname{coth}\left(d / L_{\mathrm{c}}\right)} .
$$

The distribution of volume forces acting on the lattice and caused by the external electric field, within the approximation linear in $E$, for $0<x<d$ (the linear approximation is the reason why in the equation 14 we can take into account only the part which is independent on $E$ ) is given by

$$
\begin{aligned}
f(x)=q(x) E(x)= & -\frac{\gamma e \rho_{0}\left(\mu+\varepsilon_{0}\right) E}{2 L_{\mathrm{c}} \sinh ^{2}\left(d / L_{\mathrm{c}}\right)}\left[\sinh \frac{d}{L_{\mathrm{c}}}+\sinh \frac{d-2 x}{L_{\mathrm{c}}}+\sinh \frac{2 x}{L_{\mathrm{c}}}\right. \\
& \left.-\frac{\gamma \zeta}{\sinh \left(d / L_{\mathrm{c}}\right)}\left(\frac{b}{L_{\mathrm{c}}} \sinh \frac{d}{L_{\mathrm{c}}}-1+\cosh \frac{d}{L_{\mathrm{c}}}\right) \sinh \frac{2 x}{L_{\mathrm{c}}}\right]
\end{aligned}
$$

where

$$
\gamma=\left(1+\frac{4 b}{a_{\mathrm{B}}}+\zeta \operatorname{coth} \frac{d}{L_{\mathrm{c}}}\right)^{-1}
$$
$[9]$

The stress tensor $\sigma_{x x}$ is related to the distribution of forces $f(x)$ by the equation

$$
\frac{\mathrm{d} \sigma_{x x}}{\mathrm{~d} x}+f(x)=0
$$

All other components of $\sigma_{i j}$ are zero. Making use of (15) and (16)-(17), we obtain

$$
\begin{aligned}
\sigma_{x x}(x)=-\int_{0}^{x} f(x) \mathrm{d} x= & \frac{\gamma e \rho_{0}\left(\mu+\varepsilon_{0}\right) E}{4 \sinh ^{2}\left(d / L_{\mathrm{c}}\right)}\left[\frac{2 x}{L_{\mathrm{c}}} \sinh \frac{d}{L_{\mathrm{c}}}+\cosh \frac{d}{L_{\mathrm{c}}}\right. \\
& \left.-\cosh \frac{2 x-d}{L_{\mathrm{c}}}+(1-p)\left(\cosh \frac{2 x}{L_{\mathrm{c}}}-1\right)\right],
\end{aligned}
$$

where we denote

$$
p=\frac{\gamma \zeta}{\sinh \left(d / L_{\mathrm{c}}\right)}\left(\frac{b}{L_{\mathrm{c}}} \sinh \frac{d}{L_{\mathrm{c}}}-1+\cosh \frac{d}{L_{\mathrm{c}}}\right) .
$$


The maximum stress arises in the region $d<x<d+b$, where it does not depend on $x$, because the bulk charge is absent inside this region. The corresponding value is determined by equation (18) with $x=d$

$$
\sigma_{x x}^{(\max )}=\frac{\gamma e \rho_{0}\left(\mu+\varepsilon_{0}\right) E}{2}\left[\left(\frac{d}{L_{\mathrm{c}}}+\gamma \zeta\right)+1-\frac{\gamma \varsigma b}{L_{\mathrm{c}}}-\gamma \zeta \operatorname{coth}\left(\frac{d}{L_{\mathrm{c}}}\right)\right] .
$$

From this follows the expression for the deformation tensor (within the isotropic continuum approximation [9])

$$
u_{i j}=\frac{1}{\widetilde{E}}\left[(1+\sigma) \sigma_{i j}-\sigma \sigma_{i j} \delta_{i j}\right]
$$

where $\widetilde{E}$ is the elasticity module, and $\sigma$ is the Poisson factor. Using (19)-(20), we find

$$
\begin{gathered}
u_{x x}=\frac{\gamma e \rho_{0}\left(\mu+\varepsilon_{0}\right) E}{2 \widetilde{E}}\left[\left(\frac{d}{L_{\mathrm{c}}}+\gamma \zeta\right)+1-\frac{\gamma \zeta b}{L_{\mathrm{c}}}-\gamma \zeta \operatorname{coth} \frac{d}{L_{\mathrm{c}}}\right] \\
u_{y y}=u_{z z}=-\frac{\gamma e \rho_{0}\left(\mu+\varepsilon_{0}\right) \sigma E}{2 \widetilde{E}}\left[\left(\frac{d}{L_{\mathrm{c}}}+\gamma \zeta\right)+1-\frac{\gamma \zeta b}{L_{\mathrm{c}}}-\gamma \zeta \operatorname{coth} \frac{d}{L_{\mathrm{c}}}\right] .
\end{gathered}
$$

Equations (23) and (24) give us the maximum value of deformations produced by the external electric field in the spacer region.

\section{Discussion}

Within the framework of our simplified model we have found the nonzero components of the deformation tensor depending on the applied electric field $E$ and on the geometrical parameters of the structure. It can be a possible explanation of the piezoelectric effect in nonuniformly doped heterostructures. The obtained piezomodules correspond to $C_{3 v}$ or $C_{4 v}$ symmetry classes near the interface. (When the structure is grown in (111) direction we obtain $C_{3 v}$ and when it is grown in the (001) direction we get $C_{4 v}$ ).

There is a possibility of other explanations of the non-vanishing piezoelectricity in the structures under consideration. One of them is related to a reconstruction of the crystallic lattice in the vicinity of the interface $[10,11]$. In this case an alloy layer of lower-symmetry crystallic structure can be formed permitting a bulk piezoeffect. Our consideration shows that the effect should take place in the structure even without formating the low-symmetry crystallic structures.

Another model of piezoelectricity in superlattices has been proposed in [12]. It predicts the generation of high-frequency acoustic waves of the order of hundreds of GHz. It should be noted that this seems to be a too high value for experiments under consideration $(\sim 225 \mathrm{MHz})$. Besides, the model [12] deals with a periodical distribution of charges in the superlattice. Thus, it can not be directly applied to the heterostructure with a separate QW and a region of doped semiconductor like in the cited experiments. 
There is also a model of piezoelectricity in semiconductor superlattices [13] referring to the charge-density domain motion.

It is hard to compare deformation values for the aforementioned models because there were no numerical estimations for them. For our model, from the equation (23) and for the bulk value of SiGe elasticity module, we have obtained that the value of deformations may be up to $0.1 \%$ of the layer thickness.

We believe that a rather simple theoretical explanation of the piezoelectric effect in non-periodical heterostructures presented here can still be valid even in the case when some other mechanisms like those cited above are involved.

\section{Acknowledgements}

This work was partially supported by the Science and Technology Center of Ukraine under Grant No. 591.

\section{References}

1. Usami N., Shiraki Y. SiGe quantum structures. - In: Mesoscopic Physics and Electronics, edited by T. Ando et al., Springer, Berlin, 1998, p. 264-271.

2. Tserback C., Polatoglou H.M., Theodorou G. Unified approach to the electronic structure of strained Si/Ge superlattices. // Phys. Rev. B, vol. 47, No. 12, p. 7104-7124.

3. O'Reilly E.P. Valence band engineering in strained-layer structures. // Semicond. Sci. Technol., 1989, vol. 4, No. 1, p. 121-137.

4. Maeda N., Saitoh T., Tsubaki K., Nishida K., Kobayashi N. Enhanced electron mobility in AlGaN/InGaN/AlGaN double-heterostructures by piezoelectric effect. // Jap. J. Appl. Phys., 1999, vol. 38, No. 7, p. L799-L801.

5. Mironov O.A., Khizhny V.I., Braithwaite G., Parker E.H.C., Phillips P.J., Whall T.E., Gnezdilov V.P. Observation of piezoelectric-like behavior in coherently strained Bdoped (100)SiGe/Si heterostructures. // J. Crystal Growth, 1995, vol. 157, p. 382385.

6. Khizhny V.I., Mironov O.A., Makarovskii O.A., Braithwaite G., Mattey N.L., Parker E.H.C., Phillips P.J. Piezoelectric effect in coherently strained B-doped (001)SiGe/Si heterostructures. // Acta Phys. Pol., 1995, vol. 88, No. 4, p. 779-782.

7. Khizhny V.I., Mironov O.A., Parker E.H.C., Phillips P.J., Whall T.E. Direct evidence for a piezoelectric effect in coherently strained SiGe/Si heterostructures. // Appl. Phys. Lett., 1996, vol. 69, No. 7, p. 960-962.

8. Landau L.D., Lifshitz E.M. Electrodynamics of Continuous Media. New York, Pergamon, 1960.

9. Landau L.D., Lifshitz E.M. Theory of Elasticity. New York, Pergamon, 1986.

10. Ourmazd A., Bean J.C. Observation of order-disorder transitions in strainedsemiconductor systems // Phys. Rev. Lett., 1985, vol. 55, No. 7, p. 765-768.

11. Tischler J.Z., Budai J.D., Houghton D.C. Ordered structures in $\mathrm{Si}_{x} \mathrm{Ge}_{1-x}$ alloy thin films. // Phys. Rev. B, 1995, vol. 51, No. 16, p. 10947-10955.

12. Quinn J.J., Strom U., Chang L.L. Direct electromagnetic generation of high frequency acoustic waves in semiconductor superlattices. // Solid State Commun., 1983, vol. 45, No. 2, p. 111-112. 
13. Schomburg E., Brandl S., Hofbeck K. et al. Generation of millimeter waves with a GaAs/AlAs superlattice oscillator. // Appl. Phys. Lett., 1998, vol. 72, No. 12, p. 14981500.

\title{
П'єзоелектричний ефект в модуляційно легованих гетероструктурах $\mathrm{p}-\mathrm{Si} / \mathrm{SiGe/(001)Si}$
}

\author{
В.К.Дугаєв ${ }^{1}$, О.А.Міронов ${ }^{2}$, С.В.Косяченко ${ }^{3}$
}

1 Чернівецький відділ Інституту матеріалознавства НАН України, 58001 Чернівці, вул. І.Вільде, 5

2 Фізичний факультет університету Уорвік, Ковентрі, CV4 7AL, Великобританія

3 Буковинський фінансово-економічний інститут, 58000 Чернівці, вул. Штерна, 1

Отримано 17 квітня 2000 р., в остаточному вигляді 31 жовтня $2000 \mathrm{p}$.

Представлені результати розрахунків п'єзоелектричного ефекту в $\mathrm{Si} / \mathrm{SiGe}$ багатошаровій структурі з вузькою квантовою ямою і товстим шаром легованого напівпровідного кремнію Si. Запропонована теорія є можливим поясненням деяких недавніх екпериментів на цих структурах.

Ключові слова: гетероструктури SiGe, п'єзоелектрика

PACS: $77.65 . L y, 85.50 .+k, 73.61 .-r$ 